\title{
SELECTIVE MAC FOR OBSTACLE AWARE CEV ENVIRONMENTAL MODEL FOR VANET (V2V)
}

\author{
Usha Rani $\mathbf{B}^{1}$ and Suraiya Tarannum ${ }^{2}$ \\ ${ }^{I}$ Department of Electrical Engineering, Visvesvaraya Technological University RRC, India \\ ${ }^{2}$ Department of Electronics and Communication Engineering, HKBK College of Engineering, India
}

\begin{abstract}
Vehicular Ad Hoc Network (VANET) adopts or resembles a similar structure of Mobile adhoc network (MANET). VANET using the IEEE $802.11 p$ standard has great potential of achieving objectives of Smart intelligent transport system (SITS) for improving transport and road safety efficiency. To guarantee QoS and provide efficient network performance, a prioritized MAC need to be designed. Many priority based MAC has been designed in recent times to improve the quality of data delivery to end user. However these do not consider the impact of environment and presence of obstacle which affects the signal attenuation at the receiver end and affecting the QoS of channel availability. To address, this work present an obstacle based radio propagation model, obstacle based CEV (City, Expressway and Village) environmental model and a selective MAC to provide QoS for different services. The proposed model efficiency is evaluated in term of throughput achieved per channel, Collison and success packet transmission. To evaluate the adaptive performance of proposed AMAC experiment are conducted under CEV environment and are compared with existing MAC NCCMA. The outcome achieved shows that the proposed model is efficient in term of reducing Collison, improving packet transmission and throughput performance considering two types of services.
\end{abstract}

Keyword:

VANET, V2V, Path Loss, DSRC, WAVE, IEEE 802.11p

\section{INTRODUCTION}

VANET offers association among the automobiles that are generally classified into following three categories such as Vehicle to Infrastructure (V2I), Vehicle to Vehicle (V2V) and Hybrid network which is a combination of V2V and V2I network. In infrastructure less based VANET the automobile behave as a as transceivers that has a capability of sending and receiving data packet without the support of RSU (Road Side Unit). In infrastructure based VANET the automobiles associate with a BS (base station) it is also known as the (RSU). V2X association is a hybrid network that adopts and uses both the prototype by providing wireless links among the automobile and the infrastructure (RSU) as well as among the automobiles. VANET provisioning various application services such as traffic conditions, safety, and transportation service etc. Different application services have different data delivery priorities i.e. data packets produced by these applications is generally delivered by allocating the priorities to each one. These data packet that is with high priority are delivered in real time condition and these packets transmitted over VANET results in packet loss, collisions and significantly affects or degrades the performance in VANET and as a result the quality experienced by the end user is affected. All these concerns can be rectified by prototyping a trustworthy MAC layer protocol which can adapt to dynamic nature of VANET application services. The scholars have improved the present
MAC 802.11 standard, namely MAC 802.11p to guarantee the QoS requirements [1]. The Fig.1 represents the V2V communication architecture.

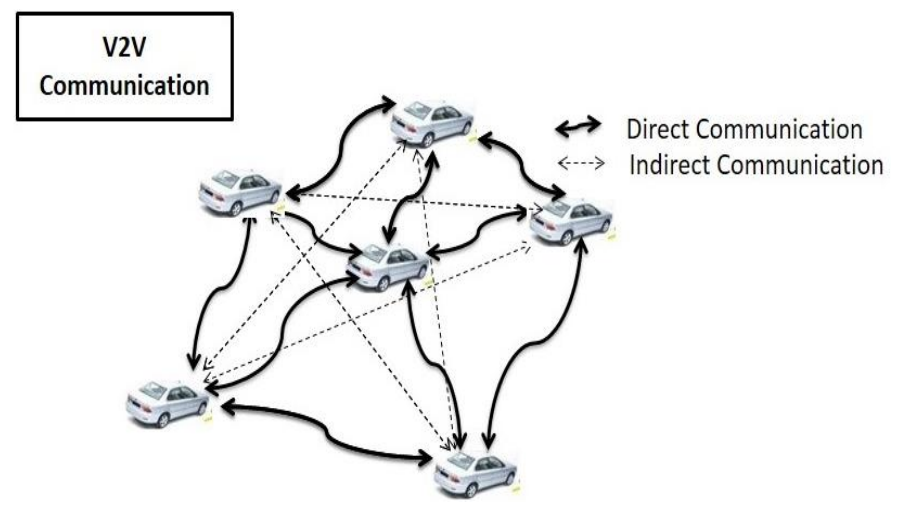

Fig.1. Architecture of V2V communication

The IEEE $802.11 \mathrm{p}$ standard was established by modifying the present IEEE 802.11a to offer the QoS assurance for the VANET based app services related to SITS, route assistance services, driver's safety and assistance and speed control administration etc. The IEEE $802.11 \mathrm{p}$ operates on the $5.9 \mathrm{GHz}$ bands and channel bandwidth of $10 \mathrm{MHz}$ [2]. The IEEE $802.11 \mathrm{p}$ adopts physical layer as association interface amid the MAC layer and the wireless links which work using OFDM (Orthogonal frequency division multiplexing) modulation. The OFDM offers channel contention access mechanism that allow devices to segment or slots the common channel [3]. The IEEE 802.11p is modulated using BPSK, QPSK, 16-QAM and 64-QAM modulation structures and the physical layer employs the 64 subcarriers and can be modulated under the constraints of channel quality [4].

VANET Application services necessitate an efficient, precise and timely conveyance of information in order to deliver comfort (such as multimedia based application services) and safety (such as vehicle tracking, ambulance services, traffic management etc.) application services and this led to the requirement of high efficiency, QoS provision and throughput necessities. Therefore designing a MAC layer play an important role in VANET automobile wireless communication. The existing work presented so far consider use of the default parameters and ideal channel conditions. The MAC can be generally classified in to following things considering the purpose of SITS based application services requirement [5]. Firstly, the schedule-based or contention-free conventions, in this kind of prototype the offer bounded access delay even in saturated (Vehicle density load is equal to the load of network capability) environments, but they necessitate reconfiguration of network parameter during network infrastructure operation in order to adjust to ever-changing network topology. Some of the MAC prototype designed of these 
conventions are as follows, CDMA (Code Division Multiple Access), FDMA (Frequency Division Multiple Access) and TDMA (Time Division Multiple Access). Secondly, the Contention-based conventions, the foremost benefit of these conventions is that they do not depend on the typical network reconfiguration exists in automobile atmospheres or VANET doesn't require reconfiguration in the conventions. Nevertheless due to the arbitrary characteristic of medium access given by these mechanisms, admittance to the medium can't be assured within a stipulated time or limit time bound. These MAC protocol is generally are nondeterministic in nature. Namely they are as follows Aloha, Slotted-Aloha, CSMA/CA and CSMA.

The IEEE 802.11p adopts CSMA/CA as its MAC mechanism. In [6] they have used EDCA (Enhanced Distributed Channel Access) in order to enhance the QoS for SITS application services. To improve the QoS considering SITS they classified the traffic in to four kinds of priorities as a result the MAC becomes contention based not the deterministic because admittance to the medium can't be assured within a stipulated time or limit time bound [7], due to this it cannot guarantee deterministic access delay. The effectiveness or impact of EDCA is influenced by priority level assigned to the data in VANET [6] [8]. Due to this priority the effectiveness of EDCA is reduced. Due to this it not efficient for SITS application which requires reliable, low-delay, reliable and real-time association is mandatory.

Considering the contention free based convention such as TDMA. Here the shared resources are separated into time slots which are assigned to every automobile or users for communication, thus helps in avoiding transmission collision. The decentralized approach such as STDMA [9] where the VANET user participant are themselves accountable for sharing the association or transmission channel and the synchronization between the devices is done through a global navigation satellite network, such as GPS (Global positioning system) due to the decentralized network topology adopted here. The drawback of this is that it needs time synchronization and also the channel access schedules are reconfigured in a periodic manner even when a scenario of satisfactory balance has been reached. To address this time synchronization problem in [10] VeSOMAC is prototyped by having bounded maximum delay with the cost of requiring periodic signaling. The applications are designed based on vehicle localization as in STDMA [9] although here it does not require time synchronization thus eliminating the likelihood of collisions and schedules are reconfigured only when the VANET infrastructure detects a conflict between automobile users over the slot selected.

The other contention-based convention is Slotted-Aloha [11] though this protocol employs conceptions from TDMA prototype but they differ in the situation of collision i.e. the selection of association times is based on a random process which means that maximum delay is not bounded for all situations. When considering topology changes for CSMA/CA the Slotted-Aloha prototype doesn't need signaling or reconfiguration, the drawback of this protocol is that collision level is high and it is not suitable for high priority services. In order to guarantee bounded medium access delay for SITS based application services there a several cognitive radio $\mathrm{CR}$ base MAC model that have been proposed such as NCCMA [12], here by applying CR techniques every automobile user stores information about the occupancy estimation of each slot. This info was then adopted to find the best slot to perform the data communication needs for various application services. Consequently that info was updated depends on the outcome of every individual communications by applying a bonuses-and-penalties procedure or methodology and this approach was a non-deterministic mechanism that enabled each individual to learn and adapt to rapid changes in that exist in VANET atmospheres. The NCCMA prototype has been designed in response to the necessities for an enhancement in the application of Dynamic Spectrum Access (DSA) and CR techniques to enhance the performance of SITS. Though this protocol has reduced the collision in the VANET they did not consider the environment factor such as path loss and shadowing, Doppler effects etc. and its impact on the network throughput.

To address these here in this work the author consider on all these issues like environment condition, traffic density or limited bandwidth, different condition of city, expressway or village area author proposed a model which includes all these things. Here author first propose a dynamic environmental model for varied environmental condition such as City (C), Express-Way (E) and Village (V), for $\mathrm{V} 2 \mathrm{~V}$ environment considering the geographical and environmental factor. Secondly the author proposes an adaptive MAC Scheduler by optimizing the beaconing scheduling and wave service advertisement in control channel for prioritized multi-channel DSRC based V2V communication in IEEE 802.11p MAC protocol considering the CEV environment model.

The paper organization is as follows: The literature survey is presented in Section 2. The proposed environmental model considering environmental factor are presented in Section 3. The results and the experimental study are presented in the section four. The concluding remark is discussed in the last section.

\section{LITERATURE SURVEY}

Every technology has some strength and weakness in terms of reliability, effectiveness, efficiency, cost, maintenance and ease of use. The primary objective of almost all the vehicular wireless technology is to provide safety and reliable service to its end user. Some of the existing methodology that has been proposed to addresses various issues pertaining to VANET such as routing, environment factor, link status; MAC based routing protocol etc. is researched below.

In [13] they did the performance evaluation considering different routing protocol of various categories of proactive (OLSR), reactive (AODV), and hybrid (ZRP). They considered various constraints such by varying the size of packet, density of mobile devices and considered various modulation schemes and used Nakagami propagation model, with the mobility of device equal to $10 \mathrm{~m} / \mathrm{s}$ for each scenario. The back-off time for all case is kept as a default constant that exists in IEEE 802.11p MAC. The simulation outcome obtained by them shows that ZRP had overall achieved a significant performance when compared to OLSR and AODV assuming the adopted conditions. This paper is an extension of our previous work.

In [14] they have analysed the significance of modulation in wireless networks. They have used QPSK and BPSK modulation for evaluation. Moreover to check the impact of error rate, SNR, data rate by adopting different modulation scheme here they have done compared the performance among between QPSK and BPSK 
modulation schemes and there result obtained shows considering the different types of topology the QPSK appeared to be performing better but they have not used QAM modulation scheme for their simulation. The QPSK will not guarantee a QOS for high throughput application which is need of hour to address this QAM can offer QOS to obtain for high throughput application services.

In [15] they evaluated some of the path loss model that has been used for VANET environment. The evaluated path loss model of their work is Nakagami and Two Ray Ground model. They have used OLSR and AODV as their routing protocol and they have considered a case of city model. The simulation performance parameter considered in their work is packet transmission end to end delay and packet delivery ratio. The performance of these two simulation metric are evaluated by varying number of connection and number of devices or automobiles for both Nakagami and Two Ray Ground model. The simulation result obtained by them shows that the Nakagami is dynamic, more realistic and it work well for VANET environment.

In [1] they addressed the issue related to broadcasting of message delay in control channel to cater this they proposed a methodology that adopts the mechanism of both TDMA and CSMA for simultaneous channel switching and scheduling. Their methodology adopted will provide efficient broadcasting due to fact they are modelled to share the service control and the intervals channel control intervals. The simulation conducted by them and their outcome shows that its performance better some of the existing MAC based scheduling protocol such as IEEE MAC, VeMAC and SOFT MAC etc. in terms of maximum throughput, collision rate and minimum. Their work that they have presented for $\mathrm{V} 2 \mathrm{~V}$ can be extended to RSU infrastructure service environment.

In [16] they have used proposed a back-off methodology to improve the throughput and channel usage efficiency and to overcome issues related to packet of packet in MANET. In [17] they have discovered various issues and challenges that exist and affect the MAC layer performance in VANET based application services. The VANET based application services are broadly classified based on their functionality and requirements and the application service are as follows such as traffic management application services, user applications services, safety application services etc. Each application services require QoS necessities and as a result different class of application services QoS is impacted due to the inefficiency of modelling the performance of MAC Layer. To study the impact performance of MAC layer for different application services here they studied various MAC based protocol such as contention free, contention based and Hybrid MAC protocol which is a combination of both contention free and contention based protocol.

In [18] they have analysed the functionality and characteristic of some of the existing routing protocol such as proactive and reactive routing protocols. Here they have considered these two routing protocol for 802.11p MAC based and used varied propagation model or scenario and also considered varied mobility speed of devices. They adopted an agent based model were the PBC (periodic broadcast) agents propagates the safety information or packet over VANET. They conducted simulation study and the outcome shows that it could not able to satisfy the QoS requirement of VANET application service. The QoS parameter considered here are Load, delay, jitter etc. and their proposed methodology induces high network overhead for VANET and as a result it is not efficient and practical for real time VANET based application service and these issues need to be addressed.

Based on the literature review it is clear that the environmental conditions and optimizing the MAC scheduler for beacon scheduling in control channel in IEEE 802.11p MAC protocol are critical to achieve an efficient SITS application service for VANET.

\section{ADAPTIVE MAC SCHEDULAR FOR CEV ENVIROMENT MODEL FOR V2V IN VANETS}

This work presents an adaptive MAC for authors CEV environment [19]. The CEV environment model presented in [19] is designed using mean attenuation model (log-normal path loss model) which do not consider the effect of presence of obstacle in $\mathrm{CEV}$ radio propagation environment modelling. The presence of obstacle between transmitter and receiver affects the signal attenuation among them. This results in drop in network performance (increases collision). Here we present an obstacle based radio propagation model. The model compute the additional attenuation required for transmission between transmitter and receiver in presence of obstacle. Then we present a selective MAC considering two types of services which is adaptive considering CEV environment. Likelihood of selectivity for channel contention for positive transmission considering two types of services is presented.

Let consider that set of vehicle $M$ passing through a CEV VANET environment i.e. $M=(1, \ldots, j)$ where each vehicle $j$ has a radio transmission range $T_{j}$. The vehicle can communicate with each other and no hidden nodes are considered i.e. each vehicle has information of all vehicle in network. The vehicle can associate with one vehicle at a time and the number of adjacent device it can communicate with at a given time $t$ can be given as:

$$
N_{i}(t)=\{(j \mid i, j) \in E(t)\}
$$

The neighboring vehicle are separated by distance $p$ from each other and the number of neighbouring vehicle which is set distance $p$ apart at instance time $i$ is obtained as follows:

$$
N_{i}(t, p)=\left\{(j \mid i, j) \in E(t), d_{i j}=p\right\}
$$

where, $d_{i j}$ is distance between automobile $i$ and $j$. The overall neighbor of a vehicle which is separated by distance $p$ can be computed as follows:

$$
f(p)=\frac{1}{T} \sum_{t=1}^{T} \frac{1}{|V(t)|} \sum_{i=1}^{|V(t)|}\left|N_{i}(t, p)\right|
$$

where, $|V(t)|$ is the total density of vehicle in the VANET environment at instance $t$.

The experimental model is designed in such way any vehicle in network can communicate with each other and transmit packet of size $O$. Each vehicle gain access to channel and access channel for stipulated session. The bandwidth of channel varies with respect to time and the user has to decide when to transmit packet based on channel availability. The channel bandwidth depends on mobility of vehicles and its environmental characteristics and the 
radio propagation model. The average arrival rate of vehicle in CEV environment can be obtained as follows,

$$
\delta=|V(t)| s
$$

where, $s$ is the speed of vehicle. The speed of vehicle in CEV environment depends on density of vehicle and can be expressed as follows

$$
s=S\left(1-\frac{|V(t)|}{|V(t)| \uparrow}\right)
$$

where, $S$ is a free flow speed of vehicle in CEV environment without presence of other vehicle in the network.

The maximum neighbor $a$ particular vehicle $b$ can accommodate is obtained as follows

$$
N_{\uparrow, b}=\left|2 A_{b|V(t)|_{\uparrow}}\right|
$$

The bandwidth of a channel at time instance $t$ can be obtained as follows,

$$
d_{t}=D \log 2\left(1+\frac{P}{N_{o} D n_{t}^{\gamma}}\right)
$$

where, $P$ is power required for transmission, $N_{o}$ is the spectral density power, $n_{t}$ is distance between transmitter and the receiver where $\gamma$ is the path loss parameter and $D$ is the bandwidth of channel.

The radio propagation signal attenuation are affected by environmental factors such shadowing, path loss and fading. The outcome obtained in [20] shows the log-normal path loss is efficient and is easy to implement which is adopted in model presented in [19]. However these models are not efficient, since these models do not estimate effects of signal attenuation in presence of obstacle in Line of Sight (LOS) between transmitter and the receiver. In [21] showed that the presence of obstacle in LOS between transmitter and receiver affect the signal attenuation at the receiver. To address the addition attenuation requirement for effective transmission, here we model the obstacle in our modelling to evaluate the $\gamma$ in Eq.(4). To compute the likelihood of LOS $(\gamma)$ between transmitting $(T)$ vehicle a and receiving $(R)$ vehicle $b$, with one nodes as probable obstacle between $T$ and $R$ (of altitude $A_{a}$ and $A_{b}$, respectively), we obtain

$$
L\left(\gamma \mid A_{a}, A_{b}\right)=1-Q\left(A-\frac{\alpha}{\delta}\right)
$$

where,

$$
A=\left(A_{a}-A_{b}\right) \frac{K_{o}}{K}+A_{a}-0.6 M_{F}+A_{x}
$$

where, $A$ represent real altitude of straight line that connects $T$ and $R$ considering the obstacle is in first Fresnel ellipsoid. The $\alpha$ depicts the mean altitude of obstructing device, $Q(\cdot)$ represent the $Q$-function and $\delta$ is the standard deviation of the obstructing device, $K_{o}$ distance between sender and the obstructing device, $K$ is the distance among $T$ and $R, A_{x}$ is the antenna altitude and $M_{F}$ is the first Fresnel ellipsoid zone radius which is computed as follows,

$$
A_{x}=\sqrt{\frac{A K_{o}\left(K-K_{o}\right)}{K}}
$$

where, $\lambda$ represent the wavelength as defined in DSRC operating in $5.9 \mathrm{GHz}$ frequency bands. If the intermediate device obstructs the first Fresnel ellipsoid by less than forty percent then the LOS is considered to be unobstructed. The additional attenuation required due to presence of obstacle is computed using [22] which modelled in our simulator.

Provisioning QoS for V2V environment possess challenges. Since each application services requires different QoS requirement (i.e. for safety application packet drop is not acceptable). To provision QoS an efficient MAC designs is required. This work present a selective MAC by assigning different selectivity to different application (Note: consider two type of service classes). The selective MAC is designed considering following environmental setup. Let consider that each vehicle is equipped with single radio which access channel every 50 milliseconds i.e. switching between control and service channels. Each vehicle transmit packet in a periodic manner which is represented as Wave short message, advertisement and beaconing message. The radio status of vehicle can be one of the following states such as idle, positive or negative. Let consider two frame type of service $X$ and $Y$. The service $X$ and $Y$ is distinguished in modelling as follows, $B_{X}$ and $B_{Y}$ is the length of frame of service $X$ and $Y$ respectively, $R_{X}$ and $R_{Y}$ is the minimum size of contention window of service $X$ and service $Y$ respectively, $K_{X}$ and $K_{X}$ is the number of vehicle of service $X$ and service $Y$ respectively and $R I F D_{X}$ and $R I F D_{Y}$ is the Random Interframe Delay (RIFD) of service $X$ and $Y$ respectively. The service $A$ is consider to have higher channel access selectivity and at the same time if service $B$ want to access channel it has wait for certain time which is computed as follows,

$$
\mu=\frac{\left(R F I D_{X}-R F I D_{Y}\right)}{\omega}
$$

where, $\omega$ is the number of slot after busy medium and it is retrieved and used for both positive and negative transmission. To cater selectivity based on frame size the back off time of service $Y$ is reduced only after waiting for $\mu$ slots and then it tries to reattempt for transmission.

The slot duration required for positive transmission of service A belong to service $c=X, Y$ is obtained as follows,

$$
Q_{t, c}=Q_{a}+\frac{F_{c}}{D}+R F I D_{X}
$$

where, $F_{c}$ is the frame size of service $c, D$ is data rate and $Q_{a}$ is time taken for convergence of physical layer. In similar way the collision or negative transmission for service $X$ is determined as follows

$$
Q_{g, c}=Q_{a}+\frac{V_{c}}{D}+R F I D_{X}
$$

The maximum time duration of collision event for service $X$, if both $X$ and $Y$ transmit is obtained as,

$$
Q_{g, \uparrow}=\uparrow\left[Q_{g, X}, Q_{g, Y}\right]
$$

The time period of Eq.(10), Eq.(13) and Eq.(14) are estimated in slots $(\omega)$ which is as follows $t_{c}=Q_{t, c} / \omega, g_{c}=Q_{g, c} / \omega$ and $g_{\uparrow}=$ $Q_{g, \uparrow} / \omega$. The individual time of each event can be computed by adding $\mu$ in the above equation since it is computed in slots at the service $B$ services. 
Let consider that the $Q_{c}$ as an important parameter for each service type in defining control channel slot duration which can be expressed as follows,

$$
\left.Q_{c}=\left(Q_{z}-Q_{m}-Q_{a}-F_{c} / D\right)\right) / \mu
$$

where, $Q_{z}$ is the control channel time period and $Q_{m}$ is the guard interval time. In $Q_{m}$ no transmission of frame is permitted or if frame transmission $\left(Q_{c}+F_{c}+D\right)$ is greater than remaining time duration of control channel.

The main objective of proposed selective MAC is to find the likelihood of positive transmission considering two service $X$ and $Y$. The likelihood of positive transmission when there are $(f-1)$ unallocated slots and $h_{c}$ nodes of service c communicates in $v^{\text {th }}$ slot, where $h_{c} \in\left[X, h_{c}\right]$ and $c=X, Y$, i.e. at least one node from service $X$ or $Y$ communicates is computed as follows,

$$
\begin{aligned}
L(Q)= & \left(1-\frac{f^{-1}}{i_{X}}\right)^{v_{X}}\left(1-\frac{f^{-1}}{i_{Y}}\right)^{v_{Y}} \times\left(\begin{array}{l}
v_{X} \\
h_{X}
\end{array}\right)\left(\frac{1}{i_{X}-f+1}\right)^{h_{X}} \\
& \left(1-\frac{1}{i_{X}-f+1}\right)^{v_{X}-h_{X}} \times\left(\begin{array}{l}
v_{Y} \\
h_{Y}
\end{array}\right)\left(\frac{1}{i_{Y}-f+1}\right)^{h_{Y}} \\
& \left(1-\frac{1}{i_{Y}-f+1}\right)^{v_{Y}-h_{Y}}
\end{aligned}
$$

where, $Q=f, v_{X}, v_{Y}, i_{X}, i_{Y}, h_{X}, h_{Y}, i_{X}$ and $i_{Y}$ is the available window size of $X$ and $Y, v_{X}$ and $v_{Y}$ are nodes of service $X$ and $Y$ respectively, $f$ is the available slots, and $h_{X}$ and $h_{Y}$ is a node that transmit, and belong to node $v_{X}$ and $v_{Y}$ respectively.

Let consider $1 /\left(i_{c}-f+1\right)$ is the likelihood of available slots $\left(i_{c}\right.$ $-f+1)$ from $(c=X, Y)$. If $c_{X}$ or $c_{Y}$ is equal to zero then the likelihood of Eq.(16) can be re-modeled by applying Bernoulli process as follows,

$$
\begin{aligned}
L(Q)= & \left(1-\frac{f-1}{i_{X}}\right)^{v_{X}}\left(1-\frac{f-1}{i_{Y}}\right)^{v_{Y}}\left(\begin{array}{c}
v_{X} \\
h_{X}
\end{array}\right)\left(\frac{1}{i_{X}-f+1}\right)^{h_{X}} \\
& \left(1-\frac{1}{i_{X}-f+1}\right)^{v_{X}-h_{X}}\left(1-\frac{f-1}{i_{Y}}\right)^{v_{Y}}\left(1-\frac{f}{i_{X}}\right)^{v_{X}} \\
& \left(\begin{array}{l}
v_{Y} \\
h_{Y}
\end{array}\right)\left(\frac{1}{i_{Y}-f+1}\right)^{h_{Y}}\left(1-\frac{1}{i_{Y}-f+1}\right)^{v_{Y}-h_{Y}}
\end{aligned}
$$

The likelihood that a node of service $Y$ does not select one of first $f$ slots of service $X$ out of $(f-1)$ slot and $v^{\text {th }}$ slot is selected by service $X$ to transmit can be assured by term $\left(1-f / i_{Y}\right)$ when $h_{Y}=0$ and $\left(1-f / i_{X}\right)$ when $h_{X}=0$.

Now let's define the likelihood that $v$ device in CEV environment select the contention window of $x$ slot out of $(f-1)$ available slots in beginning of transmission attempt and only $h$ device communicates in $f^{\text {th }}$ slot is obtained as follows,

$$
L\left(Q^{\prime}\right)=\left(1-\frac{f-1}{i}\right)^{v}\left(\begin{array}{l}
v \\
h
\end{array}\right)\left(\frac{1}{i-f+1}\right)^{h}\left(1-\frac{1}{i-f+1}\right)^{v-h}
$$

where, $Q^{\prime}=f, v, i, h$. The average positive transmission $S^{x}\left(Q^{\prime \prime}\right)$ if $v$ vehicles of service $A$ with window size $i$ and $h$ slots available in control channel is computed as follows,

$$
\begin{aligned}
& S^{X}\left(Q^{\prime \prime}\right)=\sum_{f=1}^{\downarrow(i, q)} L(f, v, i, X)(1-B)^{i_{X}} *\left[1+S^{X}(A)\right] \\
&+ L(f, v, i, X)\left(1-(1-B)^{f_{X}}\right) \\
& * S^{X}\left(q-f+1-g_{X}, i-f, v-1\right) \\
& L(f, v, i, X)(1-B)^{i_{X}} *\left[1+S^{X}(A)\right] \\
& \sum_{f=1}^{\downarrow(i, q)} L(f, v, i, X) S^{X} \\
& \quad \times\left(q-f+1-g_{X}, i-f, g-1\right)
\end{aligned}
$$

where, $Q^{\prime \prime}=(q, i, v), \quad A=q-f+1-t_{\mathrm{x}}, \mathrm{i}-f, v-1$ is the average positive transmission in the available $\left(q-f+1-t_{X}\right)$ slots. The term one in above equation shows the likelihood that out of $v$ device only one vehicle will positively transmit data in $f^{\text {th }}$ slot by picking up back-off from $i$ slots and $S^{x}(A)$ is the average positive transmission in the available $\left(q-f+1-t^{X}\right)$ slot. The second term defines the likelihood of packet failure due to presence of noise and third term defines the collision likelihood in the $f^{\text {th }}$ slot when $i(i \geq 2)$ of $v$ vehicles communicates. Similar to Eq.(19) the average positive transmission service $Y$ is computed below,

$$
\begin{aligned}
S^{X}\left(Q^{\prime \prime}\right)= & \sum_{f=1}^{\downarrow(i, q)} L(f, v, i, X)(1-B)^{i_{X}} *\left[1+S^{X}\left(\begin{array}{c}
q-f+1 \\
-\left(t_{X}+\mu\right), \\
i-f, g-1
\end{array}\right)\right] \\
& +L(f, v, i, X)\left(1-(1-B)^{i_{Y}}\right) \\
& * S^{Y}\left(q-f+1-\left(g_{X}+\mu\right), i-f, v-1\right) \\
& \sum_{h=1}^{v} L(f, v, i, h) S^{Y} \\
& \times\left(q-f+1-\left(g_{X}+\mu\right), i-f, v-1\right)
\end{aligned}
$$

The Eq.(19) and Eq. (20) defines the likelihood of mean positive transmission $S^{x}\left(Q^{\prime \prime}\right)$ and $S^{y}\left(Q^{\prime \prime}\right)$ of service $X$ and $Y$ respectively. The likelihood parameter depends on service $Y$ back off time after busy medium as defined in Eq.(11) and the average positive transmission during control channel is computed as follows:

$$
S^{X}(Q)=\left\{\begin{array}{cc}
S^{X}(Q)\left(h, v_{x}, i_{x}\right) & v_{Y}=0 \\
0 & v_{X}=0
\end{array}\right.
$$

where,

$$
\mathrm{Q}=h, v_{\mathrm{X}}, v_{Y}, i_{X}, i_{Y}
$$

and

$$
L_{\mu\left(v_{X}, i_{X}\right)}=\left\{\begin{array}{cc}
\left(\frac{i_{X}-\mu}{i_{X}}\right)^{v_{X}} & \mu<i_{X} \\
0 & \mu \geq 0
\end{array}\right.
$$

is the likelihood that number vehicle of service $X$ in selective slots $\mu$.

Similarly likelihood of cumulative positive transmission for class $Y, S^{Y}(Q)$ can be computed. Based on this the likelihood of positive transmission for vehicle of service $c$, with $c=X, Y$ can be computed as:

$$
L^{c}=\left(S^{c}\left(Q_{X}, V_{X}, V_{Y} I_{X}, I_{Y}\right) / K_{c}\right.
$$


Here the likelihood of positive transmission considering two types of services is modelled. In our model the frame is considered to be dropped when vehicle back off counter does not decrease to zero at the end of control channel interval. Our model can also be used to compute the frame loss due to Collison and noise. In next section the experiment are conducted to evaluate the performance of Proposed CEV-AMAC and the results are compared with exiting MAC [12] [16] [9] [4]. A comparison of these existing model is shown in Table.1.

Table.1. Comparison with other works

\begin{tabular}{|c|c|c|c|c|c|}
\hline & $\begin{array}{c}\text { Proposed } \\
\text { adaptive } \\
\text { Selective } \\
\text { MAC }\end{array}$ & $\begin{array}{c}\text { ENCCM } \\
\text { A } \\
{[\mathbf{1 2}]}\end{array}$ & $\begin{array}{c}\text { ZCOR } \\
{[\mathbf{1 6}]}\end{array}$ & $\begin{array}{c}\text { EDF- } \\
\text { CSMA } \\
{[\mathbf{9}]}\end{array}$ & $\begin{array}{c}\text { CCVC } \\
{[\mathbf{4}]}\end{array}$ \\
\hline $\begin{array}{c}\text { Medium } \\
\text { Access } \\
\text { Control } \\
\text { adopted }\end{array}$ & $\begin{array}{c}802.11 \mathrm{p} \\
\text { MAC }\end{array}$ & $\begin{array}{c}802.11 \mathrm{p} \\
\text { MAC }\end{array}$ & $\begin{array}{c}802.11 \mathrm{e} \\
\text { MAC }\end{array}$ & $\begin{array}{c}802.11 \mathrm{p} \\
\text { MAC }\end{array}$ & $\begin{array}{c}802.11 \mathrm{p} \\
\text { MAC }\end{array}$ \\
\hline $\begin{array}{c}\text { Medium } \\
\text { Access } \\
\text { Control } \\
\text { scheduling } \\
\text { adopted }\end{array}$ & A-MAC & ENCCMA & $\begin{array}{c}\text { RR- } \\
\text { ALOHA }\end{array}$ & $\begin{array}{c}\text { EDF- } \\
\text { CSMA }\end{array}$ & $\begin{array}{c}\text { Slotted } \\
\text { aloha }\end{array}$ \\
\hline $\begin{array}{c}\text { Environmen } \\
\text { t Modelling }\end{array}$ & CEV & $\begin{array}{c}\text { Freely } \\
\text { flowing } \\
\text { vehicles }\end{array}$ & $\begin{array}{c}\text { Monte } \\
\text { Carlo }\end{array}$ & $\begin{array}{c}\text { No } \\
\text { access }\end{array}$ & $\begin{array}{c}\text { Realistic } \\
\text { vehicular } \\
\text { communi } \\
\text { cation }\end{array}$ \\
\hline $\begin{array}{c}\text { Simulator } \\
\text { adopted }\end{array}$ & SIMITS & SIMITS & $\begin{array}{c}\text { NS-2 } \\
\text { simulati } \\
\text { on }\end{array}$ & NS-3 & $\begin{array}{c}\text { Yes (no } \\
\text { access) }\end{array}$ \\
\hline
\end{tabular}

\section{SIMULATION RESULT AND ANALYSIS}

The system environment used is windows 10, 64-bit quad core operating system with $16 \mathrm{~GB}$ of ram and $2 \mathrm{~GB}$ dedicated. The author have used dot net framework 4.0 and $C \# 6.0$ programming language for the proposed work and conducted experimental study on following parameter for Packet transmission positively, packet collision and throughput efficiency for number of vehicle users and evaluate the performance of the proposed adaptive MAC for varied environmental model. The authors have considered IEEE 802.11p also known as DSRC/WAVE which has six services channel and one control channel and CEVAMAC as MAC scheduling algorithm.

The modulation scheme used is QAM-64 which has as a transfer rate of $27 \mathrm{Mbps}$ (Megabits per seconds) with coding rate of 0.75 and the experiment is conducted by varying the number of vehicle is to 20,40 and 80 and simulation is conducted for Village (V), Expressway (E) and City (C) environmental model and the result of proposed MAC is evaluated with existing algorithm as shown in Table.1.

\subsection{THROUGHPUT PERFORMANCE ANALYSIS}

The Fig. 2 it shows the throughput efficiency for varied vehicle in city environmental model.

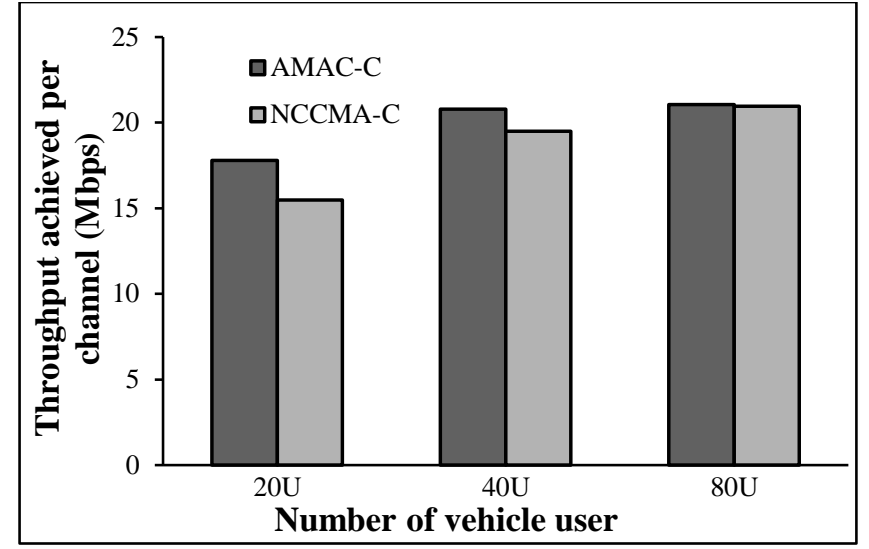

Fig.2. Throughput achieved for City environment considering varied vehicles

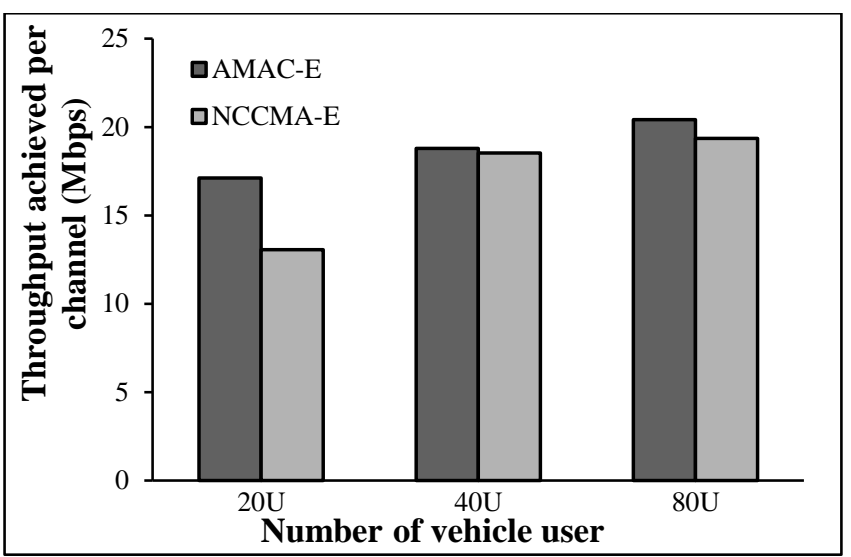

Fig.3. Throughput achieved for Expressway environment considering varied vehicles

Table.2. Comparison with other works

\begin{tabular}{|c|c|c|c|c|c|c|}
\hline & AMAC- & NCCMA- & AMAC & NCCM & AMAC & NCCM \\
C & C & -E & A-E & -V & A-V \\
\hline $\mathbf{2 0}$ & & 15.495 & 17.1167 & 13.070 & 14.7959 & 13.564 \\
Vehicle & 17.78958 & 34 & 1 & 07 & 8 & 19 \\
\hline $\mathbf{4 0}$ & & 19.508 & 18.7918 & 18.528 & 15.971 & 15.822 \\
Vehicle & 20.78539 & 12 & 5 & 14 & 90 & 89 \\
\hline $\mathbf{8 0}$ & & 20.956 & 20.4222 & 09.350 & 17.515 & 16.238 \\
vehicle & 21.05513 & 18 & 6 & 40 & 20 & 41 \\
\hline
\end{tabular}

The Fig.3 shows the throughput efficiency for varied vehicle in expressway environmental model. Fig. 4 shows the throughput efficiency for varied vehicle in village environmental model.

From all three environment model in Fig.2, Fig.3 and Fig.4 it can be seen that throughput achieved is increased with increasing number of vehicle for both the proposed AMAC and existing NCCMA algorithm [12] and the simulation value obtained in Mbps is tabulated in below Table.2. 


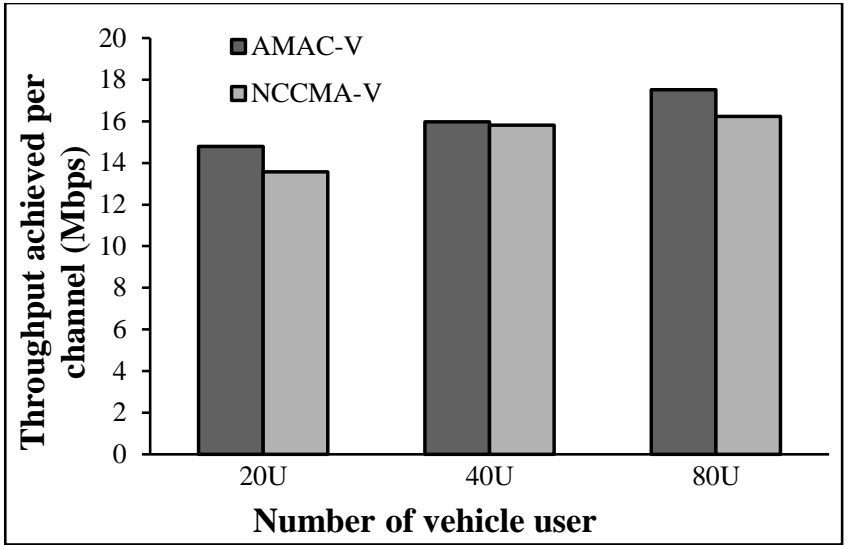

Fig.4. Throughput achieved for Village environment considering varied vehicles

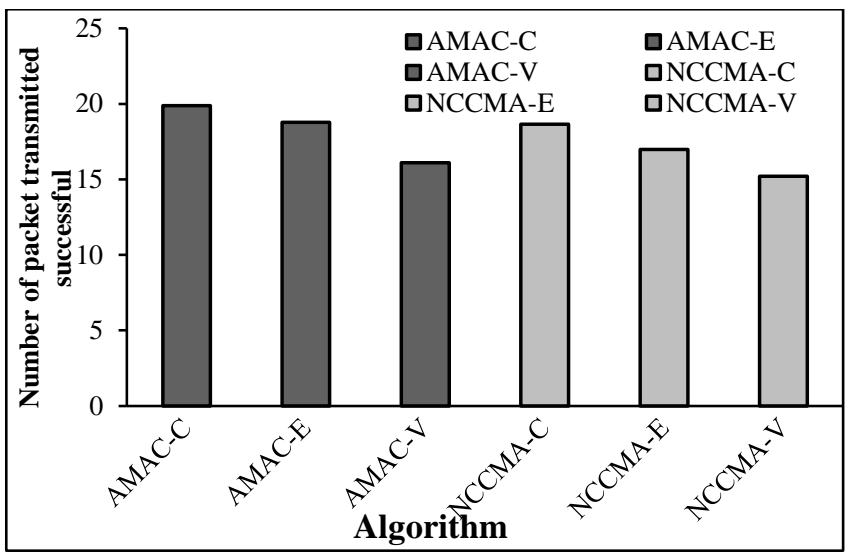

Fig.5. Average throughput achieved for CEV environment

The Fig.5 represent the achieved throughput for the different user (20, 40 and 80) for our CEV environmental model and from analysis we observed that the achieved throughput in case of city is high as compared to village and expressway. While achieved throughput for village environment is low in all. Achieved throughput for city environment is $19.876 \mathrm{Mbps}$ for proposed AMAC while for existing NCCMA it is $18.653 \mathrm{Mbps}$.

Improved throughput percentage for AMAC is $6.16 \%$ over existing NCCMA. Similarly we analysed the throughput performance for expressway and village for AMAC and NCCMA and find the throughput for expressway and village considering AMAC is 18.776 and $16.094 \mathrm{Mbps}$ respectively. While for NCCMA throughput for expressway is $16.094 \mathrm{Mbps}$ and for village it is $15.208 \mathrm{Mbps}$.

Improved throughput percentage for AMAC over NCCMA for expressway is $9.96 \%$ and for village it is $5.62 \%$. For all CEV environments the proposed AMAC perform better than Existing NCCMA in term of throughput achieved.

\subsection{COLLISION PERFORMANCE ANALYSIS}

For city, expressway and village environmental model, packet collision performance analysis is done and it is presented in Fig.6, Fig. 7 and Fig. 8 respectively.

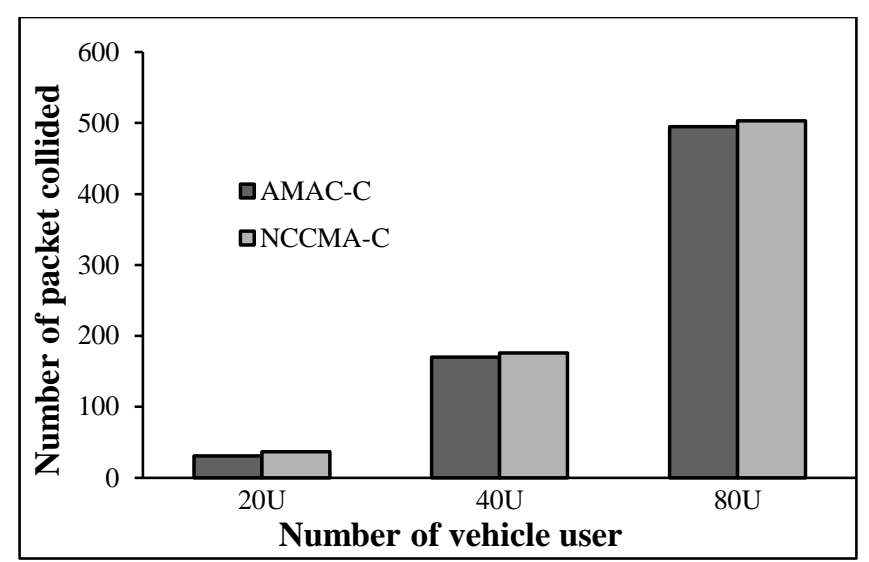

Fig.6. Packet collision analysis for City environment

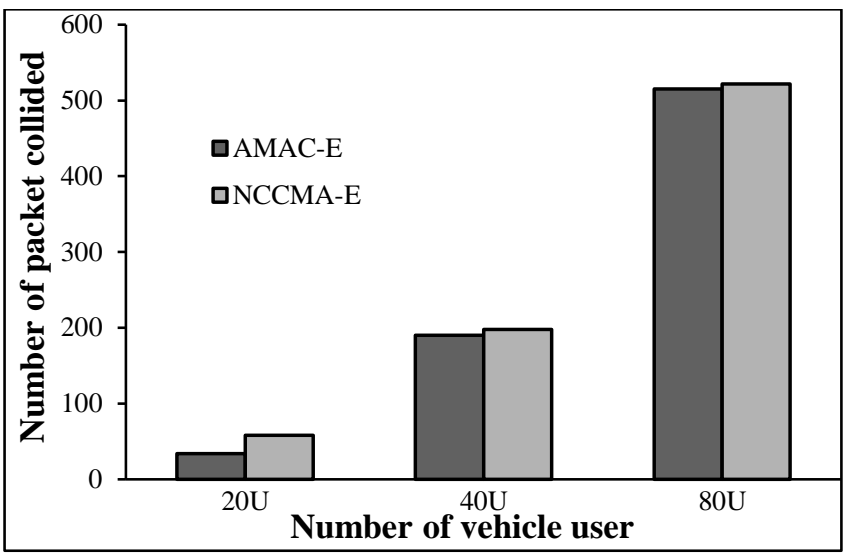

Fig.7. Packet collision analysis for Expressway environment

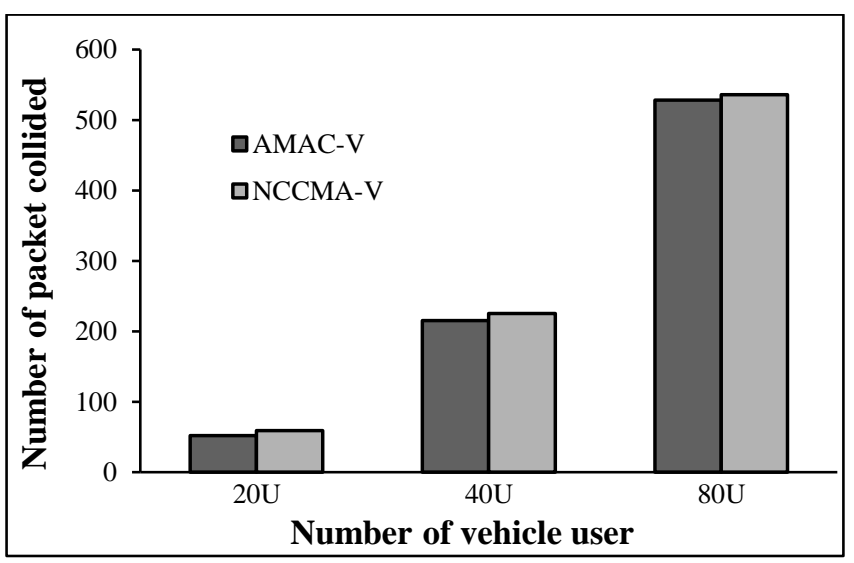

Fig.8. Packet collision analysis for Village environment

Analysis of packet collision shows that as number of vehicle increased in the network, packet collision also increased for both the existing NCCMA and proposed AMAC. Average packet collision in the network is represented in the Fig.9. From the above analysis, we can observe that the collision of packet is high for the village environment as compared to expressway and city environment, while collision of data packet observed low for the city environment as compared to other. From analysis we can also say that with increased number of user collision also get increased in the network. Packet collision reduced $2.8 \%$ for AMAC in case of city environment as compared to, similarly reduced packet collision for expressway is observed as $5.02 \%$ and for village 
environment it is $3.05 \%$ as compared with existing NCCMA. Now we are in position to tell that for all environmental model, (city, expressway and village) proposed AMAC performed better than existing NCCMA in terms of packet collision performance analysis.

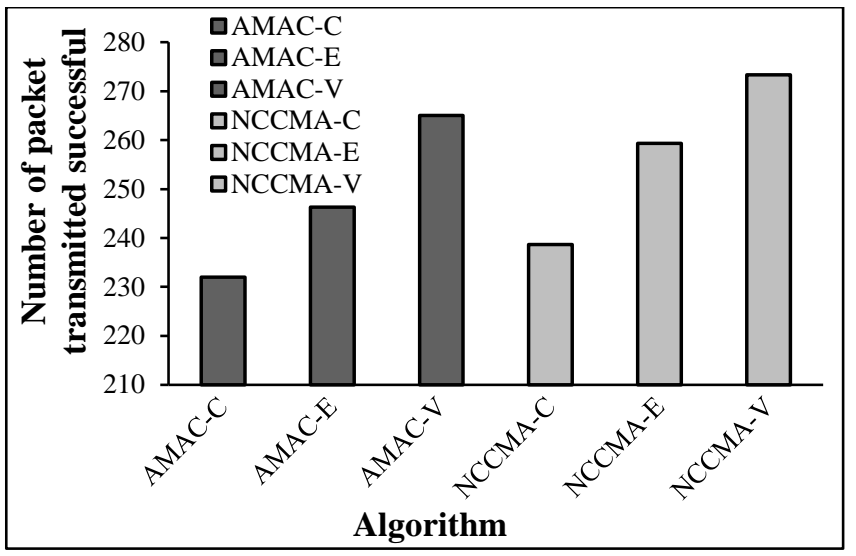

Fig.9. Packet collision analysis for CEV environment

\subsection{PACKET TRANSMISSION SUCCESSFUL ANALYSIS}

The Fig. 10 shows the packet transmission performance analysis for varied vehicle in city environmental model. The Fig. 11 shows the packet transmission performance analysis for varied vehicle in expressway environmental model. The Fig.12 shows the packet transmission performance analysis for varied vehicle in village environmental model. From all three environment model in Fig.10 to Fig.12, it can be seen that positive or successful packet transmission is increased with increasing number of vehicle for both the proposed AMAC and existing NCCMA algorithm.

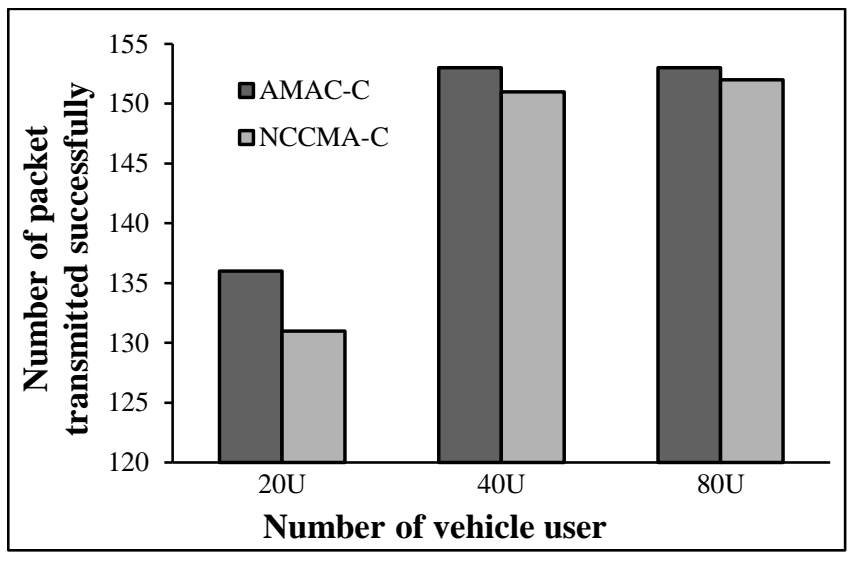

Fig.10. Packet transmission successful analysis for City environment.

The Fig.13 shows the average packet transmission in V2V. The packet transmission is high in city model when compared to expressway and village model and packet transmission is low in village model when compared to expressway and village model and also we can see that the packet transmission positively increases with increasing number of vehicles. The successful transmission of packet of the proposed AMAC for city environment is improved by $2.76 \%$ over the existing NCCMA. The successful transmission of packet of the proposed AMAC for expressway environment is improved by $8.74 \%$ over the existing
NCCMA. The successful transmission of packet of the proposed AMAC for village environment is improved by $4.43 \%$ over the existing NCCMA. For all CEV environments the proposed AMAC perform better than Existing NCCMA in term of packet transmission performance achieved.

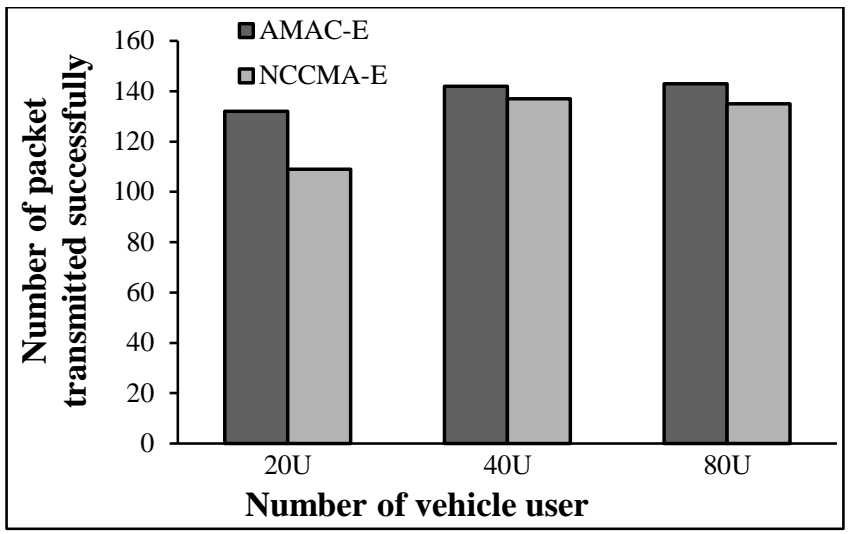

Fig.11. Packet transmission successful analysis for Expressway environment

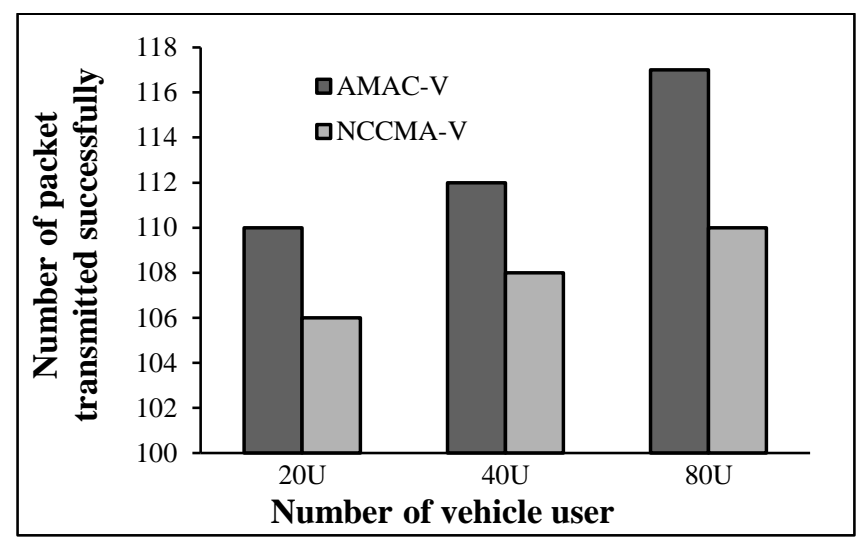

Fig.12. Packet transmission successful analysis for Village environment

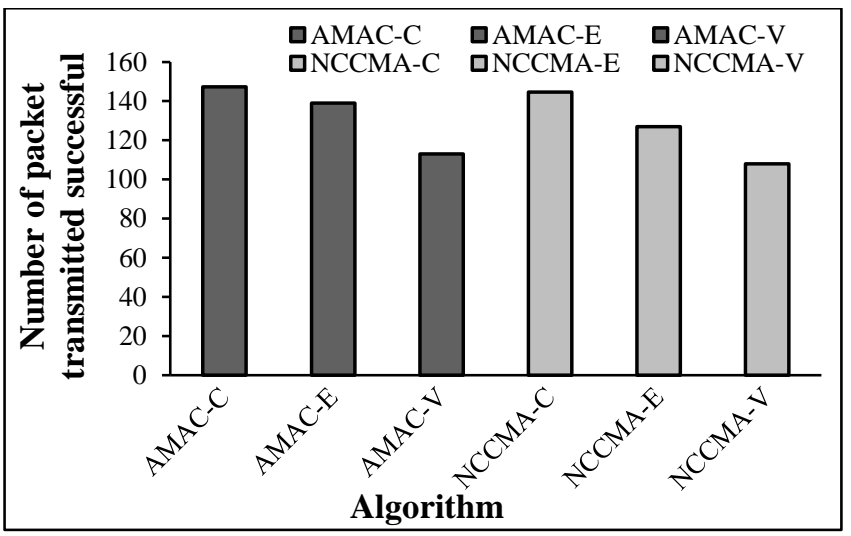

Fig.13. Average Packet transmission successful analysis for CEV environment

\section{CONCLUSION}

VANET has great potential of achieving objectives of Smart intelligent transport system (SITS) for improving transport and road 
safety efficiency. Various prioritized MAC has been designed to provision different QoS for different services. These model radio propagation are designed by adopting mean attenuation model. The mean attenuation model is not efficient considering the presence of obstacle in LOS and also considering different environment. As a result of this, the channel availability, network performance and QoS is affected. To address this work, we present a selective MAC, obstacle based radio propagation model and CEV environment model. Experiments are conducted to evaluate the performance proposed AMAC in term of packet collision, successful transmission and throughput efficiency. The experimental result shows the proposed AMAC performs better than exiting NCCMA for $\mathrm{CEV}$ environment model in terms of packet collision, positive packet transmission, throughput efficiency and thus helps in aiding improving QoS for VANET application. In future work, we would evaluate the performance of proposed AMAC considering varied mobility speed and varied packet load for CEV environmental model and evaluate the performance considering various OFDM modulation scheme such BPSK, QPSK and QAM that are available for IEEE 802.11p DSRC Protocol and discover which modulation scheme is good for VANET based SITS. Security is another factor that need be considered in designing secure VANET model.

\section{REFERENCES}

[1] L. Zhang, Z. Liu, R. Zou, J. Guo and Y. Liu, "A Scalable CSMA and Self-Organizing TDMA MAC for IEEE 802.11 p/1609.x in VANETs", Wireless Personal Communications, Vol. 74, No. 4, pp. 1197-1212, 2014.

[2] N. Bouchema, R. Naja and A. Tohme, "Traffic Modeling and Performance Evaluation in Vehicle to Infrastructure 802.11p Network", Proceedings of International Conference on Ad Hoc Networks, pp. 82-99, 2014.

[3] Mohammad M. Shurman, Mamoun F. Al-Mistarihi and Zaid A. Alomari, "MAC Layer Back-off Algorithm for Ad-Hoc Networks", Proceedings of $36^{\text {th }}$ International Convention on Information and Communication Technology Electronics and Microelectronics, pp. 23-27, 2013.

[4] S. Oh, M. Gruteser and D. Pompili, "Coordination-Free Safety Messages Dissemination Protocol for Vehicular Networks", IEEE Transactions on Vehicular Technology, Vol. PP, No. 99, pp. 1-13, 2012.

[5] R.A. Saeed, A.B. Hj Naemat, A.B Aris, I.M. Khamis and M.K. Bin-Awang, "Evaluation of the IEEE 802.11p-based TDMA MAC method for Road Side-to-Vehicle Communications", International Journal of Network and Mobile Technologies, Vol. 1, No. 2, pp. 81-87, 2010.

[6] Saurabh Sehrawat, Revoti Prasad Bora and Dheeraj Harihar, "Performance Analysis of QoS Supported by Enhanced Distributed Channel Access (EDCA) Mechanism in IEEE 802.11e", IAENG International Journal of Computer Science, Vol. 33, No. 1, pp. 1-6, 2007.

[7] Katrin Bilstrup, Elisabeth Uhlemann, Erikg Strom and Urban Bilstrup, "On the Ability of the 802.11p MAC Method and STDMA to Support Real-Time Vehicle-toVehicle Communication", EURASIP Journal on Wireless Communications and Networking, Vol. 2009, pp. 16871472, 2009.

[8] Stephan Eichler, "Performance Evaluation of the IEEE 802.11p Wave Communication Standard", Proceedings of $66^{\text {th }}$ IEEE Vehicular Technology Conference, pp. 21992203, 2007.

[9] Che-Yu Chang, Hsu-Chun Yen and Der-Jiunn Deng, "V2V QoS Guaranteed Channel Access in IEEE 802.11p VANETs", IEEE Transactions on Dependable and Secure Computing, Vol. 13, No. 1, pp. 5-17, 2015.

[10] Fan Yu and Subir Biswas, "Self-Configuring TDMA Protocols for enhancing Vehicle Safety with DSRC based Vehicle-to-Vehicle Communications", IEEE Journal on Selected Areas in Communications, Vol. 25, No. 8, pp. 1526-1537, 2007.

[11] Lawrence G. Roberts, "Aloha Packet System with and without Slots and Capture", ACM SIGCOMM Computer Communication Review, Vol. 5, No. 2, pp. 28-42, 1975.

[12] Mario Manzano, Felipe Espinosa, Angel M. Bravo-Santos and Alfredo Gardel-Vicente, "Cognitive Self-Scheduled Mechanism for Access Control in Noisy Vehicular Ad Hoc Networks", Mathematical Problems in Engineering, Vol. 2015, pp. 1-12, 2015.

[13] Aarja Kaur and Jyoteesh Malhotra, "On the Selection of QoS Provisioned Routing Protocol through Realistic Channel for VANET", International Journal of Scientific and Technology Research, Vol. 4, No. 7, pp. 191-196, 2015.

[14] A.U. Haque, M. Saeed and F.A. Siddiqui, "Comparative Study of BPSK and QPSK for Wireless Networks over NS2", International Journal of Computer Applications, Vol. 41, No. 19, pp. 8-12, 2012.

[15] Pranav Kumar Singh, "Influences of Two Ray Ground and Nakagami Propagation Model for the Performance of Adhoc Routing Protocol in VANET", International Journal of Computer Applications, Vol. 45, No. 22, pp. 1-6, 2012.

[16] Haiyue Piao, Yongtae Park, Byungjo Kim and Hyogon Kim, "Safety Beaconing Rate Control based on Vehicle Counting in Wave", Proceedings of IEEE Intelligent Vehicles Symposium, pp. 1-6, 2015.

[17] S.A. Gillani, P.A. Shah, A. Qayyum and H.B. Hasbullah, "MAC Layer Challenges and Proposed Protocols for Vehicular Ad-hoc Networks", Vehicular Ad-hoc Networks for Smart Cities, Vol. 306, pp. 3-13, 2015.

[18] Md Habibur Rahman and Mohammad Nasiruddin, "Impact of Two Realistic Mobility Models for Vehicular Safety Applications", Proceedings of International Conference on Informatics, Electronics and Vision, pp. 1-6, 2014.

[19] B. Usha Rani and Suraiya Tarannum, "A Channel Modelling considering Varied Environmental model for DSRC based V2V Application Services", Proceedings of $3^{\text {rd }}$ International Conference on Devices, Circuits and Systems, pp. 48-53, 2016.

[20] Sayyid A. Vaqar and Otman Basir, "Traffic Pattern Detection in a Partially Deployed Vehicular Ad Hoc Network of Vehicles", IEEE Wireless Communications, Vol. 16, No. 6, pp. 40-46, 2009.

[21] W. Viriyasitavat, M. Boban, H.M. Tsai and A. Vasilakos, "Vehicular Communications: Survey and Challenges of Channel and Propagation Models", IEEE Vehicular Technology Magazine, Vol. 10, No. 2, pp. 55-66, 2015.

[22] International Telecommunication Union, "Propagation by Diffraction", Available at: https://www.itu.int/rec/R-RECP.526/en. 